\title{
The long non-coding RNA and mRNA expression profiles in keratinocytes from patients with psoriasis vulgaris
}

\author{
Yilun Wang", Junhao Zhu\#, Jinhua Xu, Juan Du, Xiaonian Lu \\ Department of Dermatology, Huashan Hospital, Fudan University, Shanghai, China \\ Contributions: (I) Conception and design: J Du, Y Wang; (II) Administrative support: X Lu, J Xu; (III) Provision of study materials or patients: X Lu, \\ J Du; (IV) Collection and assembly of data: J Zhu; (V) Data analysis and interpretation: Y Wang; (VI) Manuscript writing: All authors; (VII) Final \\ approval of manuscript: All authors. \\ \#These authors contributed equally to this work. \\ Correspondence to: Juan Du; Xiaonian Lu. Department of Dermatology, Huashan Hospital, Fudan University, 12 Wulumuqi Zhong Road, Shanghai \\ 200040, China. Email: dendritic_jj@163.com; 13816830396@139.com.
}

\begin{abstract}
Background: Psoriasis is a chronic inflammatory dermatosis. The hyperproliferation and hyperkeratosis of keratinocytes is a key step in the pathogenesis of psoriasis. Long non-coding RNAs (lncRNAs) and mRNAs regulate gene expression in various biological process, including the function of keratinocytes. This research investigated the expression profile of lncRNAs and mRNAs in keratinocytes of patients with psoriasis vulgaris.

Methods: The expression of lncRNAs and mRNAs in keratinocytes from patients with psoriasis vulgaris and healthy patients was examined and compared using microarrays. Quantitative polymerase chain reaction (qPCR) and bioinformatic analysis was also performed. DAVID and KEGG were used to analyze the gene function. The competing endogenous RNA (ceRNA) network was also constructed.

Results: A total of 48 lncRNAs and 17 mRNAs were differentially expressed in keratinocytes of psoriasis vulgaris. Quantitative PCR data showed that the expression of lnc-AGXT2L1-2:2 (P=0.009) and NR_027032 $(\mathrm{P}=0.033)$ was up-regulated in psoriasis vulgaris. The lncRNA-miRNA-mRNA interaction network was established. The mRNA showing the most connections with the lncRNAs and miRNAs was CEP104. The miRNA showing the most connections with the lncRNAs and mRNAs was miR-484. The lncRNA showing the most connections with miRNAs and mRNAs was ENST00000494887.
\end{abstract}

Conclusions: The identification of the differentially expressed lncRNAs and mRNAs in psoriasis vulgaris provides significant insights into the pathogenesis of the disease.

Keywords: Expression profile; keratinocytes; long non-coding RNA (lncRNA); mRNA; psoriasis vulgaris

Submitted Jul 02, 2021. Accepted for publication Aug 19, 2021.

doi: 10.21037/apm-21-2046

View this article at: https://dx.doi.org/10.21037/apm-21-2046

\section{Introduction}

Psoriasis is a chronic inflammatory dermatosis. Due to the unknown etiology and lack of effective treatments, the course of the disease is generally prolonged and recurring, and significantly affects the quality of life of these patients (1). The incidence and prevalence vary greatly between different ethnic backgrounds and geographic regions. In China, psoriasis affects approximately
$0.1 \%$ to $0.5 \%$ of the population (2). Psoriasis can be divided into four subtypes based on the clinical characteristics, namely, psoriasis vulgaris, psoriasis arfhropathica, psoriasis pustulose, and erythrodermic psoriasis. Psoriasis vulgaris is the most common clinical type and accounts for about $85-90 \%$ of all cases (3).

The pathogenesis of psoriasis is complex. The disease is specific genetic susceptible and characterized by various exogenous or endogenous stimulation signals. It is generally 
Table 1 The clinical characteristics of patients with psoriasis vulgaris

\begin{tabular}{lcc}
\hline Gender & Age & PASI \\
\hline Female & 27 & 36 \\
Female & 40 & 35 \\
Male & 33 & 41.4 \\
Female & 48 & 25.2 \\
Male & 58 & 24 \\
Male & 21 & 16.8 \\
Male & 35 & 49.9 \\
Male & 54 & 53.9 \\
Male & 40 & 29.7 \\
Male & 52 & 14.4 \\
Male & 53 & 23.4 \\
Female & 45 & 44.1 \\
Female & 42 & 21.6 \\
Male & 26 & 21 \\
Female & 64 & 36 \\
\hline PASl, psorasis & 5 & \\
\hline
\end{tabular}

PASI, psoriasis lesion area and severity score.

believed that the abnormal activation of immunocytes, especially $\mathrm{T}$ cells, is involved in the pathogenesis of psoriasis. The infiltration of immunocytes within the skin triggers the expression of cytokines and induces the abnormal proliferation and activation of keratinocytes (4). The resultant associated skin damage is characteristic of psoriasis vulgaris patients

During various biological processes, non-coding RNAs, especially long non-coding RNAs (lncRNAs), can act as modulators of gene expression. Recent studies have shown that non-coding RNAs are key to the occurrence and development of psoriasis through regulating the expression of important proteins. The non-coding RNA PRINS mediates abnormal keratinocyte proliferation in patients with psoriasis by regulating the expression of the G1P3 protein (5). In addition, microRNA(miRNA)31 promotes keratinocyte proliferation by inhibiting protein phosphatase 6 (6). In 2018, Qiao et al. found that lnc-MSX2P1 promoted the growth and proliferation of keratinocytes through the suppression of miR-6731-5p and the activation of the $\mathrm{S} 100 \mathrm{~A} 7$ gene. It was suggested that the lnc-MSX2P1-miR-6731-5p-S100A7 axis participates in the abnormal proliferation of keratinocytes in psoriasis (7). All these studies suggested that non-coding RNAs are closely related to the abnormal proliferation of keratinocytes. The different categories of non-coding RNAs are not isolated, and can form a complex regulatory network to participate in disease regulation. Therefore, elucidating the role of noncoding RNAs in psoriasis will be beneficial in improving the understand of the pathogenesis of the disease. This current study examined the IncRNA and mRNA expression profiles of keratinocytes in patients with psoriasis vulgaris. We present the following article in accordance with the MDAR reporting checklist (available at https://dx.doi.org/10.21037/ apm-21-2046).

\section{Methods}

\section{Patients}

A total of 15 patients with progressive psoriasis vulgaris were recruited from Huashan Hospital, Fudan University, China. The healthy skin control group consisted of 15 agematched and gender-matched patients who underwent plastic surgery in the same hospital . Patients with psoriasis did not take internal medicine for 3 months and did not use external drugs for 1 month prior to this study. The psoriasis lesion area and severity score (PASI) of patients with psoriasis is listed in Table 1. All procedures performed in this study involving human participants were in accordance with the Declaration of Helsinki (as revised in 2013). The study was approved by the Medical Ethics Committee of Huashan Hospital (NO.: 2019-541) and informed consent was taken from all the patients.

\section{Human epidermal keratinocyte culture}

Skin samples were acquired from the skin lesions of participants with psoriasis vulgaris. Negative controls were acquired from the healthy skin of patients who underwent plastic surgery. The skin samples were incubated overnight at $4{ }^{\circ} \mathrm{C}$ in Dispase solution (Roche life science products). The epidermis was separated using the trypsin digestion method. Keratinocytes were cultured in keratinocyte serumfree complete medium. The second or third generation of keratinocytes was used for RNA isolation.

\section{$R N A$ isolation and quantitative analysis}

Total RNA was isolated and purified using the Qiagen Kit (Germany). Quantitative reverse transcription (RT)polymerase chain reaction (PCR) was performed according 
Table 2 The primer sequences used in the quantitative polymerase chain reactions

\begin{tabular}{lc}
\hline Primer Name & Sequence (5' to 3') \\
\hline Actin-F & ACCATTGGCAATGAGCGGTT \\
Actin-R & GCGGATGTCCACGTCACACT \\
Inc-AGXT2L1-2:2-F & TATAACTGCTATTCTTGCAGCCCTT \\
Inc-AGXT2L1-2:2-R & TCCCATAGGACCATCTGTACCC \\
NR_027032-F & TCAAAGTTTTCCTCTGAGTGG \\
NR_027032-R & AAGCAGGTAACAAGTGGGGA \\
NR_004407-F & AACTCGATTGCTCTGCGTGC \\
NR_004407-R & CACCAGCTGCCCAAATACCA \\
ENST00000528514-F & AAGGTTGGCTTCAGAACTGG \\
ENST00000528514-R & GTTCCCGTTGGTITTCTTG \\
\hline
\end{tabular}

to the manufacturer's instructions. The primers are listed in Table 2.

\section{LncRNA and mRNA microarray}

LncRNA and mRNA microarrays were performed according to the manufacturer's protocols (Agilent technologies, Santa Clara, US). All data has been deposited into the Gene Expression Omnibus (GEO) with accession number GSE146149.

\section{Analysis of gene function}

Gene molecular function was analyzed through the Database for Annotation, Visualization and Integrated Discovery (DAVID). The roles of the genes in the pathways were analyzed by the Kyoto Encyclopedia of Genes and Genomes (KEGG) database.

\section{Construction of the competing endogenous RNA (ceRNA) network}

Data regarding miRNAs related to the differentially expressed lncRNAs and mRNAs was downloaded from miRBase using miRanda software.

\section{Statistical analysis}

The Student's $t$-test was applied for statistical analyses and $\mathrm{P}<0.05$ was considered statistically significant.

\section{Results}

\section{The expression profile of lncRNAs in keratinocytes of patients with psoriasis vulgaris}

The expression profile of lncRNAs in keratinocytes obtained from psoriasis vulgaris patients was compared to that of healthy controls using a genome-wide analysis. Hierarchical cluster analysis revealed significant differences in the expression of 48 lncRNAs (including 9 upregulated lncRNAs and 39 downregulated lncRNAs) in the keratinocytes of psoriasis vulgaris patients and healthy patients (fold change $\geq 1.5 ; \mathrm{P}<0.05$; Figure $1 A$ ). The 48 differentially expressed lncRNAs were divided into 6 classes, including 23 intergenic lncRNAs, 11 exonic-sense, 6 exonic-antisense, 4 intronic-sense, 2 intronic-antisense, and 2 bidirectional lncRNAs (in total online: https://cdn. amegroups.cn/static/public/apm-21-2046-1.xlsx).

\section{The expression profile of mRNAs in keratinocytes of patients with psoriasis vulgaris}

Significant differences were identified in the expression of 17 mRNAs (fold change $\geq 1.5, \mathrm{P}<0.05$ ) in psoriasis vulgaris keratinocytes compared with healthy keratinocytes (Figure $1 \mathrm{~B}$ ), including 3 up-regulated mRNAs and 14 downregulated mRNAs (in total online: https://cdn. amegroups.cn/static/public/apm-21-2046-2.xlsx). Gene ontology (GO) analysis revealed that the differentially expressed mRNAs could be classified into three domains. In the biological process domain, the top three GO terms were cation transport, ion transport, and small molecule metabolic process (Figure $2 A$ ). In the molecular function domain, the top three GO terms included organic cyclic compound binding, nucleic acid binding, and small molecule binding (Figure 2B). The top three GO terms in the cellular component domain were intracellular, intracellular organelle, and cytoplasm (Figure 2C). KEGG pathway analysis was performed to identify the important signaling pathways and the interactions associated with the 17 differentially expressed mRNAs. There were 11 signaling pathways enriched in keratinocytes of patients with psoriasis vulgaris. The top 5 pathways identified were mismatch repair, African trypanosomiasis, malaria, aminoacyl-tRNA biosynthesis, and antigen processing and presentation (Figure 2D).

\section{Confirmation of the differentially expressed lncRNAs}

Four differentially expressed lncRNAs were randomly 
A

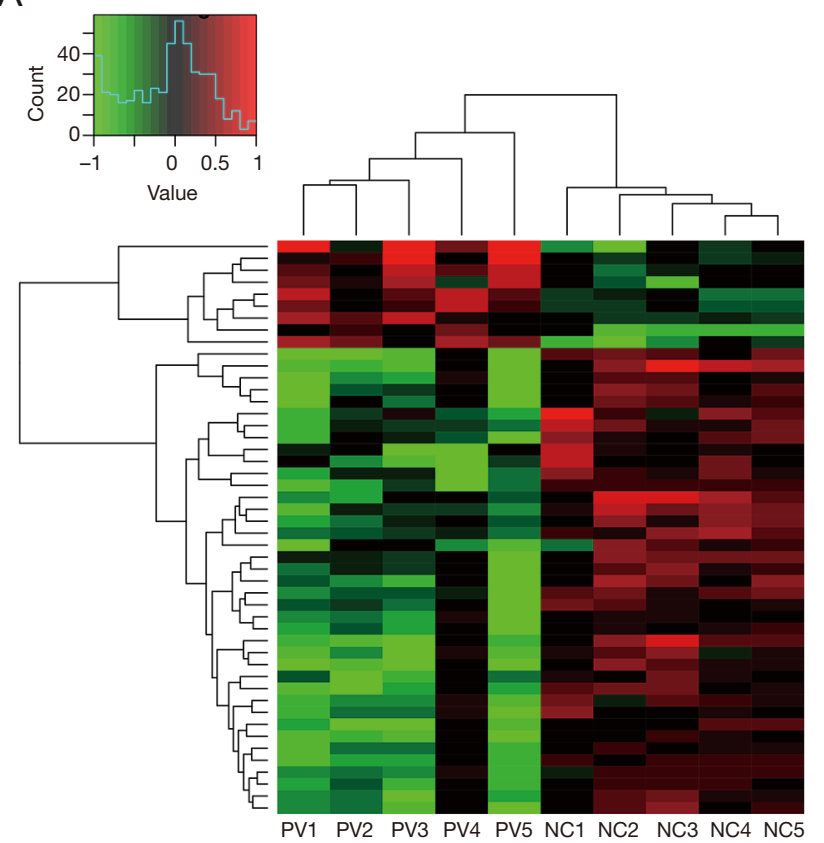

B

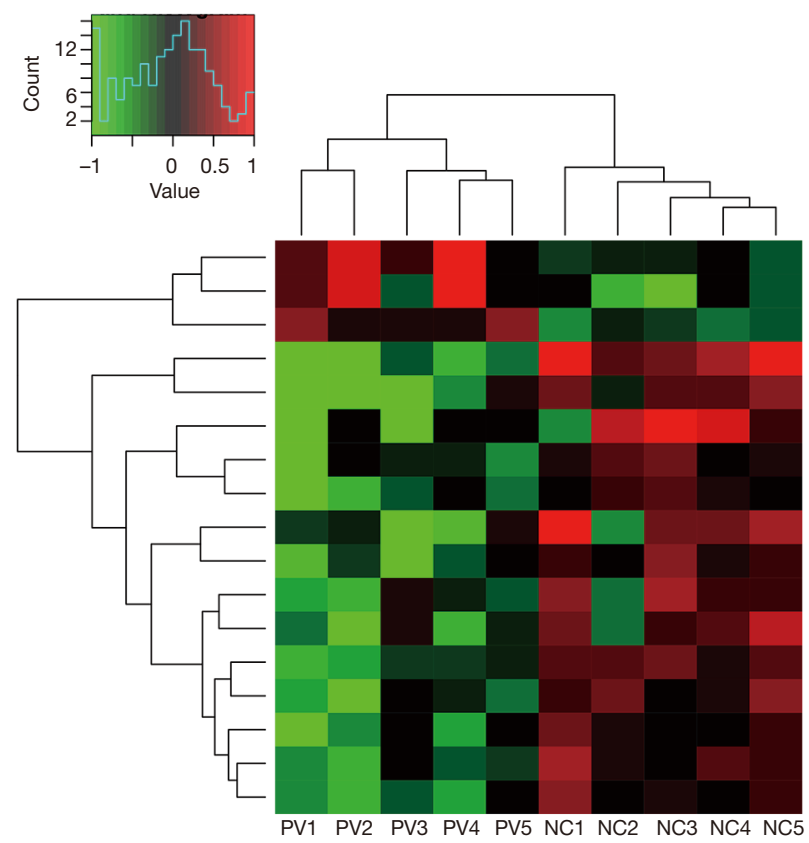

Figure 1 Hierarchical clusters of differentially expressed long non-coding RNAs (lncRNAs) and mRNAs. (A) Significant differences in the expression of 48 lncRNAs ( 9 upregulated lncRNAs and 39 downregulated lncRNAs) were found in psoriasis vulgaris keratinocytes ( $\mathrm{n}=5$ ) compared with normal controls $(\mathrm{n}=5$ ) (fold change $\geq 1.5$; $\mathrm{P}<0.05$ ). (B) Significant differences in the expression of $17 \mathrm{mRNAs}$ (fold change $\geq 1.5 ; \mathrm{P}<0.05)$ were identified in psoriasis vulgaris keratinocytes $(\mathrm{n}=5)$ compared with healthy controls $(\mathrm{n}=5)$, including 3 up-regulated mRNAs and 14 downregulated mRNAs (fold change $\geq 1.5 ; \mathrm{P}<0.05$ ). In $(\mathrm{A})$ and $(\mathrm{B})$, relatively high expression is indicated by red shading and relatively low expression is indicated by green shading. NC1-5 represents normal controls. PV1-5 represents patients with psoriasis vulgaris.

selected for qRT-PCR analysis to verify the reliability of the microarray results. The expression of lnc-AGXT2L1-2:2 and NR_027032 was up-regulated in keratinocytes from psoriasis vulgaris patients compared to healthy keratinocytes , which was consistent with the results of the microarray. However, there were no statistically significant differences in the expression of NR_004407 nor ENST00000528514 between psoriasis vulgaris keratinocytes compared to healthy keratinocytes, although there was a similar trend to that observed in the microarray analysis (Figure 3).

\section{LncRNA-target gene regulatory network analysis}

Possible lncRNA “cis” genes are defined as chromosomal co-expression genes less than $10 \mathrm{~kb}$ upstream and downstream of the differentially expressed lncRNAs. In total online: https://cdn.amegroups.cn/static/public/apm21-2046-3.xlsx shows the results of the "cis" analyses. The Basic Local Alignment Search Tool (BLAST) was used to select complementary or similar sequences of the differentially expressed lncRNAs. RNAplex was used to calculate the complementary energy between the 2 sequences to select Energy value $\leq-30$ sequences as transassociated genes. In total online: https://cdn.amegroups.cn/ static/public/apm-21-2046-4.xlsx shows the results of the "trans" analyses.

\section{Construction of the ceRNA network}

CeRNA analysis is a novel method for predicting the functions of lncRNAs. The miRNAs that can combine with both the differentially expressed lncRNAs and mRNAs were selected from miRBase. The ceRNA analysis provided an overview of the potential lncRNA-miRNAmRNA interactions (Figure 4). The results showed that the ceRNA network was made up of 50 network nodes and 70 connections between 25 lncRNAs, 18 miRNAs, and 7 mRNAs. The mRNA showing the most connections with the lncRNAs and miRNAs in the co-expression network was CEP104. The miRNA showing the most connections 
A

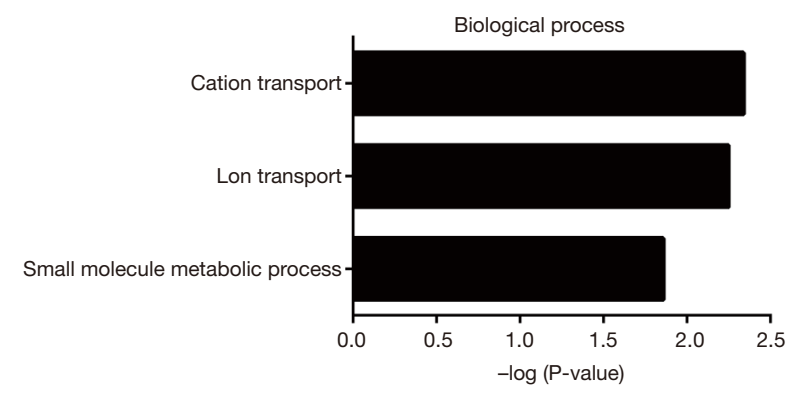

C

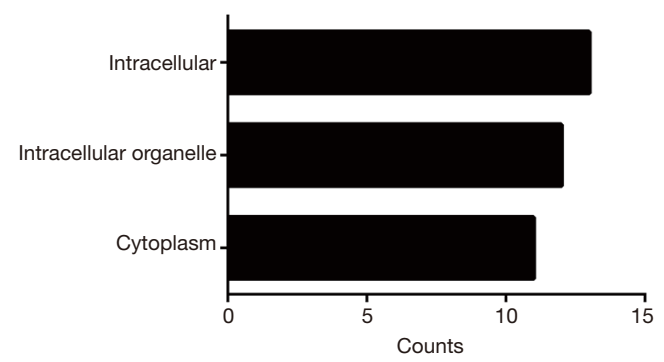

B

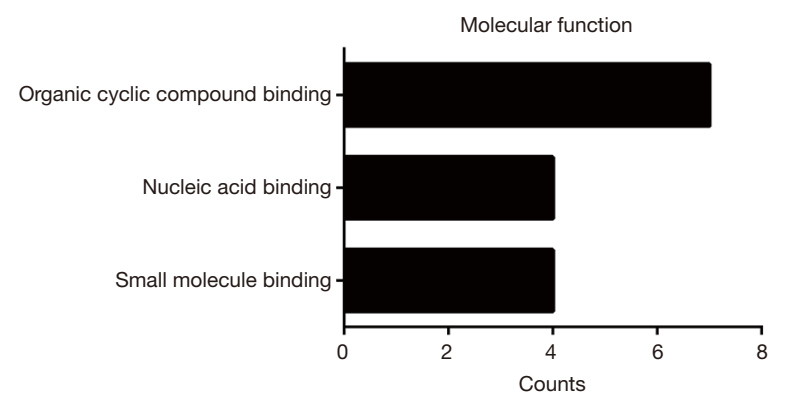

$\mathrm{D}$

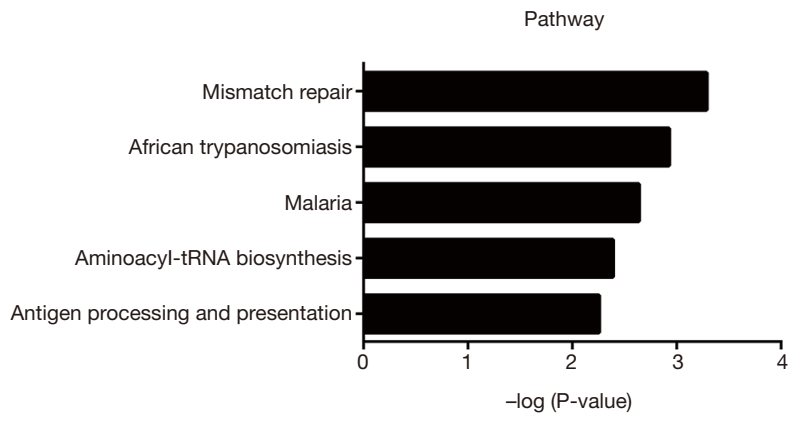

Figure 2 Gene ontology (GO) and pathway enrichment analysis for differentially regulated mRNAs. (A) The top three GO terms in the biological process. (B) The top three GO terms in the molecular function. (C) The top three GO terms in the cellular component. (D) The top five pathways of differentially expressed mRNAs in keratinocytes of patients with psoriasis vulgaris.

A

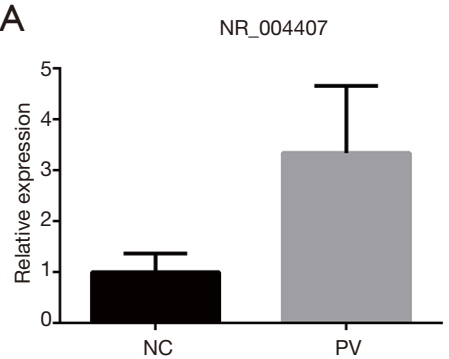

C

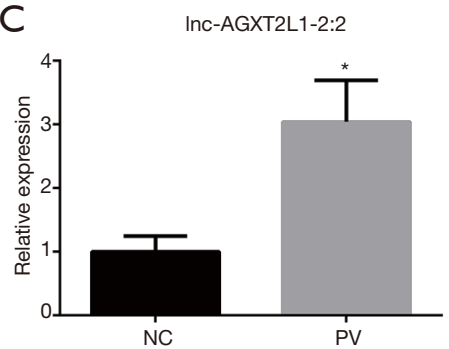

B ENST00000528514

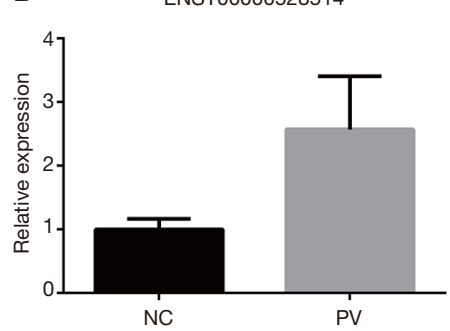

D NR_027032

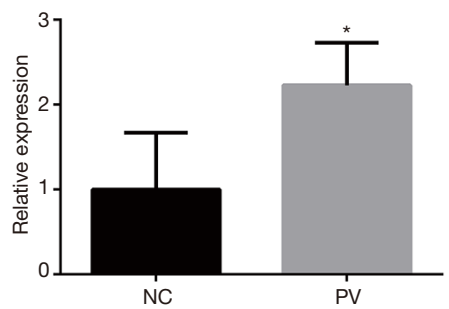

E

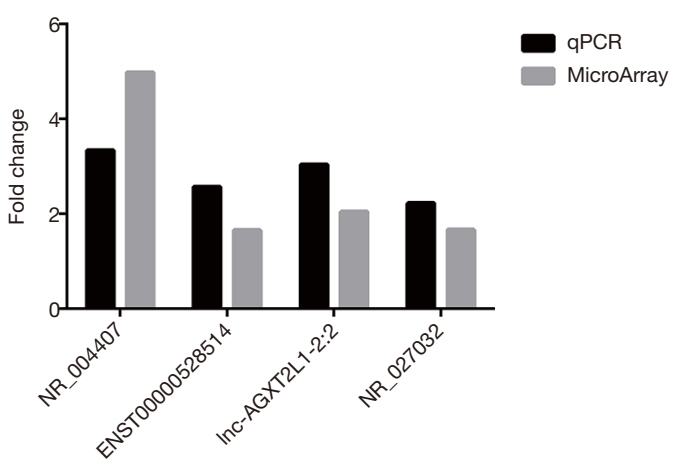

Figure 3 Confirmation of the selected long non-coding RNAs (lncRNAs) through qRT-PCR. The expression levels of (A) NR_004407 $(\mathrm{P}=0.108)$, (B) ENST00000528514 ( $\mathrm{P}=0.083)$, (C) lnc-AGXT2L1-2:2 ( $\left.{ }^{*} \mathrm{P}=0.009\right)$, and (D) NR_027032 ( $\left.{ }^{*} \mathrm{P}=0.033\right)$ in keratinocytes of normal controls (NCs) and patients with psoriasis vulgaris (PV). Data is represented as mean \pm standard deviation. (E) The fold change in expression obtained with microarray analysis and qRT-PCR. 


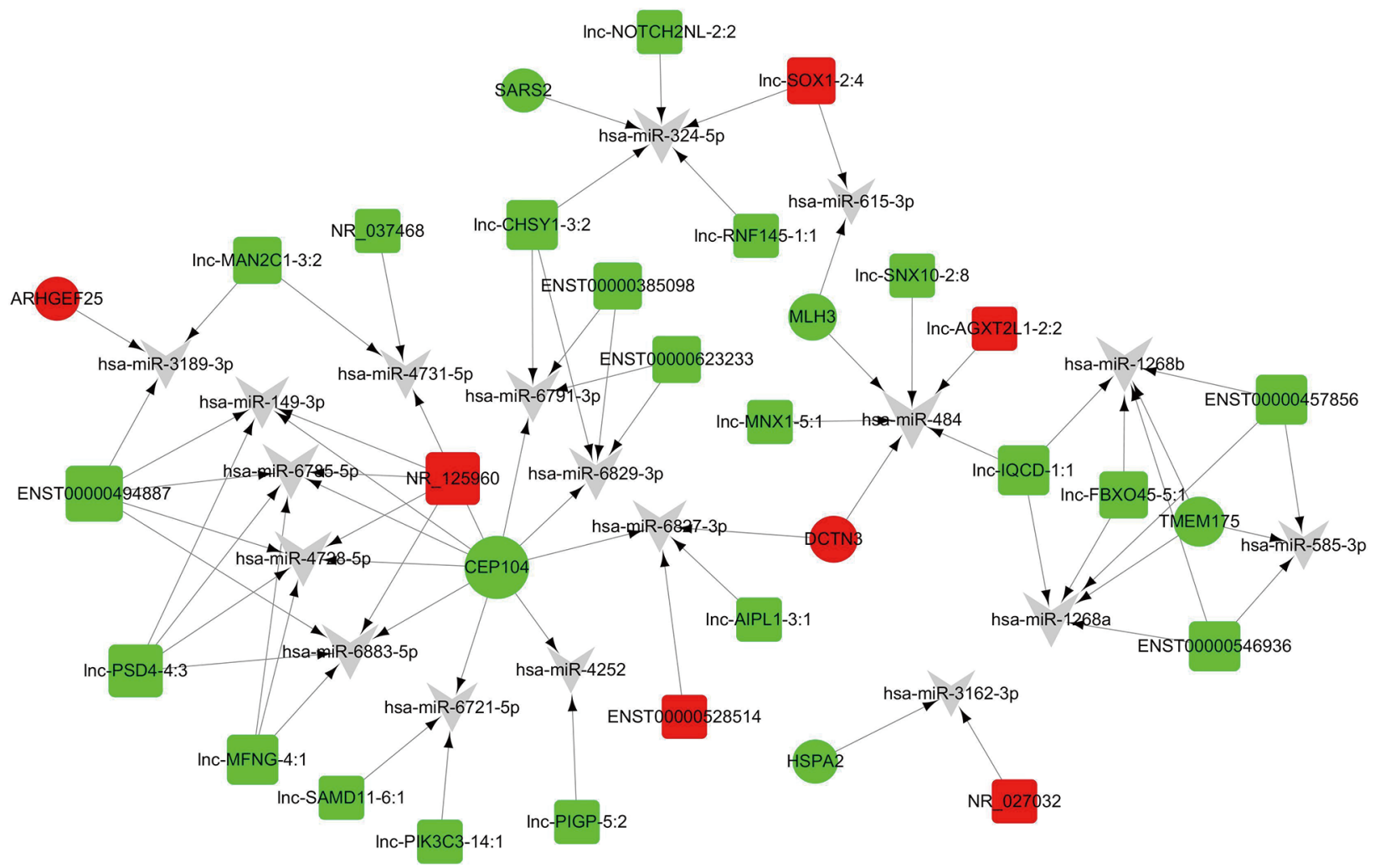

Figure 4 A ceRNA network for long non-coding RNA (lncRNA)-miRNA-mRNA in patients with psoriasis vulgaris. Triangles represent miRNAs, green circles represent down-regulated mRNAs, red circles represent up-regulated mRNAs, green squares represent downregulated lncRNAs, and red squares represent up-regulated lncRNAs.

with the lncRNAs and mRNAs was miR-484. The lncRNA showing the most connections with miRNAs and mRNAs was ENST00000494887.

\section{Discussion}

Psoriasis is a common inflammatory skin disease that decreases the quality of life of approximately 120 million people worldwide (8). The prevalence varies between different peoples and regions. In 2017, Michalek et al. summarized 76 studies in more than 20 countries and regions and revealed that the incidence of psoriasis is about $0.51 \%$ to $11.43 \%$ in adults, and $0 \%$ to $1.37 \%$ in children (9). The prevalence of psoriasis in China increased from $0.12 \%$ in 1984 to $0.47 \%$ in 2012 (10), suggesting that the incidence of psoriasis in China is escalating.

The etiology and pathogenesis of psoriasis vulgaris is complex, and is yet to be fully elucidated. The disease is specific genetic susceptible and characterized by various exogenous or endogenous stimulation signals. Activation of the signaling pathway between keratinocyte growth factor (KGF) and KGF receptor (KGFR) leads to the excessive activation and proliferation of keratinocytes in psoriasis. After binding to the receptor on keratinocytes, interleukin (IL)-21 continuously activates the STAT3 pathway through the ERK pathway, while IL-22 continuously activates the STAT3 pathway through the ERK and JNK pathways, resulting in excessive or incomplete keratinization, epidermal process extension, and loss of granular layers $(11,12)$. Keratinocytes then secrete a large number of cytokines and chemokines, including IL-17, IL-21, IL-22, interferon (IFN)-gamma and tumor necrosis factor (TNF)alpha, which could abnormally activate T-helper (Th) 1 and Th17 cells (13). The cascade amplification cross-talk between keratinocytes, $\mathrm{T}$ cells, dendritic cells, and other immune cells is the key factor in triggering and maintaining the pathological characteristics of psoriasis vulgaris (14). Keratinocytes can also be induced by activated $\mathrm{T}$ cells to produce adhesion molecules, synergistic stimulators, 
other inflammatory factors (such as IL-1 and IL-6), and vascular endothelial growth factor (VEGF) which promotes angiogenesis, thereby aggravating psoriasis (15).

In addition to genetic susceptibility, environmental factors have also been associated with psoriasis vulgaris. Non-coding RNAs, including miRNAs and lncRNAs, are RNA molecules that do not encode proteins but have various biological functions. LncRNAs, with a length of more than 200 nucleoside acids, can regulate related genes at the level of chromatin modification, transcription, and post-transcriptional processing (16). LncRNAs can interact with target mRNAs by base-pairing to either increase or suppress translation in the cytosol. They can also regulate signaling pathways via RNA-protein interactions. In the nucleus, lncRNAs can interact with specific proteins to act as a guide, decoy, or scaffold (17).

To date, many studies have focused on differentially expressed lncRNAs in the blood of patients with psoriasis, but few reports have focused on lncRNAs in the epidermis of psoriasis patients, especially within the skin lesion. Tsoi et al. used RNA-seq data from 99 lesional psoriatic, 27 uninvolved psoriatic, and 90 normal skin biopsies to detect 2,942 previously annotated and 1,080 novel lncRNAs which are expected to be skin specific (18). Yan et al. identified 2,194 lncRNAs and 1,725 mRNAs that were significantly dysregulated in the skin tissue of psoriasis patients (19). However, these studies did not report on cell-specific lncRNAs within the skin. In our current study, we identified 48 lncRNAs and 17 mRNAs that were differentially expressed in skin keratinocytes of patients with psoriasis vulgaris and healthy patients. These results suggested that the imbalances in epigenetic networks may be involved in psoriasis and keratinocytes may be important regulators. Recent studies showed that IncRNAs, such as the lncRNA psoriasis-susceptibility-related RNA gene induced by stress (PRINS), can contribute to psoriasis (5). PRINS is a transcript that is highly expressed in psoriatic non-lesional epidermis and can alter the cellular stress response (20). The deregulation of PRINS may result in suppressed sensibility to spontaneous keratinocyte apoptosis under the modulation of G1P3 $(5,21)$. However, to date, the precise roles of lncRNAs in psoriasis remain unclear.

This current investigation identified 48 lncRNAs, including lncRNA-AGXT2L1, and 17 mRNAs, including NAP1L1, that were differentially expressed in the results of the microarray. GO and KEGG pathway analyses suggested that these mRNAs were involved in cation transport, mismatch repair, and antigen processing and presentation. NAP1L1, which was part of the nucleosome assembly protein family, regulates numerous pathophysiological processes by affecting chromatin agglutination or nucleosome formation. NAP1L1 is involved in cell proliferation and differentiation $(22,23)$, tumor development and metastasis (24), inflammation and infection (25), as well as cell proliferation. A recent study showed that lncRNA-CDKN2B-AS1 can promote NAP1L1-mediated PI3K/AKT/mTOR signaling pathway through "molecular sponge" absorption of miRNA let-7c$5 \mathrm{p}$, thus leading to proliferation of liver cells (26). In our study, NAP1L1 expression was elevated in the keratinocytes of psoriasis vulgaris patients, suggesting that NAP1L1 may be involved in the proliferation of keratinocytes in psoriasis. The precise relationship between NAP1L1 and psoriasis warrants further investigation.

To verify the data of the microarrays, qPCR was performed in 15 patients and 15 healthy controls. The expression of lnc-AGXT2L1-2:2 and NR_027032 was elevated in psoriasis vulgaris patients compared to healthy controls, and this agreed with the microarray data. The expression of NR_004407 and ENST00000528514 detected by qPCR showed a similar trend to that observed with microarray analysis, however, the differences were not statistically significant. Some factors may have affected the results of the microarray. The small sample size in the microarray may lead to false positive results, and thus, the sample size was expanded in the $\mathrm{qPCR}$ analyses. There were also some technical limitations with the microarray, such as cross-hybridization, signal saturation, and limited dynamic range. Future studies should increase the sample size to further verify these results. To investigate the potential role of lnc-AGXT2L1-2:2 and NR_027032 in psoriasis, knockdown or overexpression experiments should be performed in the future.

Bioinformatics was used to predict complex interactions between the lncRNAs and miRNAs that were enriched in psoriasis skin lesions. ceRNAs interact with each other by competitively binding to shared miRNAs. Prediction of ceRNA cross-talk therefore relies on the identification of miRNA response elements (MREs) (27). Qiao et al. demonstrated that the lnc-MSX2P1-mir-6731$5 \mathrm{p}-\mathrm{S} 100 \mathrm{~A} 7$ axis participates in the abnormal proliferation of keratinocytes in psoriasis, suggesting that the ceRNA mechanism plays a part in the regulation of psoriasis. In our ceRNA network, miR-484 was the miRNA most connected by lncRNAs and mRNAs. Previous studies have shown that miR-484 can suppress translation of mitochondrial fission 
protein Fis1, and inhibit Fis1-mediated fission and apoptosis in the adrenocortical cancer cells (28). Combined with the information from the miRBase functional database, miR484 may be closely related to the abnormal proliferation of cells. It also has co-regulatory interactions with lncAGXT2L1-2:2. Further investigations examining the role of the lnc-AGXT2L1-2:2- miR-484-mRNA axis in psoriasis is warranted.

Keratinocytes play important roles in skin damage in psoriasis vulgaris patients. The findings presented in this report provide insights into the hyperproliferation and hyperkeratosis of keratinocytes, and the pathogenesis of psoriasis in an epigenetic way.

\section{Conclusions}

The study demonstrated the expression profiles of lncRNAs and mRNAs in keratinocytes of patients with psoriasis vulgaris. Differentially expressed lncRNA-target gene regulatory networks and ceRNA networks were constructed. The differentially expressed lncRNAs and mRNAs may participate in the pathogenesis of psoriasis vulgaris and provide novel insights into potential treatments.

\section{Acknowledgments}

Funding: This research was supported by the National Natural Science Foundation of China (Grant No. 81673081 and 82003382) and the Natural Science Foundation of Shanghai (19ZR1407600). Persons associated with the funding agencies played no role in the design of the study, data collection and analysis, nor preparation of the manuscript.

\section{Footnote}

Reporting Checklist: The authors have completed the MDAR reporting checklist. Available at https://dx.doi. org/10.21037/apm-21-2046

Data Sharing Statement: Available at https://dx.doi. org/10.21037/apm-21-2046

Conflicts of Interest: All authors have completed the ICMJE uniform disclosure form (available at https://dx.doi. org/10.21037/apm-21-2046). The authors have no conflicts of interest to declare.
Ethical Statement: The authors are accountable for all aspects of the work in ensuring that questions related to the accuracy or integrity of any part of the work are appropriately investigated and resolved. All procedures performed in this study involving human participants were in accordance with the Declaration of Helsinki (as revised in 2013). The study was approved by the Medical Ethics Committee of Huashan Hospital (NO.: 2019-541) and informed consent was taken from all the patients.

Open Access Statement: This is an Open Access article distributed in accordance with the Creative Commons Attribution-NonCommercial-NoDerivs 4.0 International License (CC BY-NC-ND 4.0), which permits the noncommercial replication and distribution of the article with the strict proviso that no changes or edits are made and the original work is properly cited (including links to both the formal publication through the relevant DOI and the license). See: https://creativecommons.org/licenses/by-nc-nd/4.0/.

\section{References}

1. Boehncke WH, Schön MP. Psoriasis. Lancet 2015;386:983-94.

2. Zhao Y, Lai W. Patient considerations and targeted therapies in the management of psoriasis in Chinese patients: role of ustekinumab. Patient Prefer Adherence 2014;8:865-72.

3. Grozdev I, Korman NJ. Psoriasis: Clinical Review and Update. Advances in Psoriasis 2014:21-6. doi:10.1007/9781-4471-4432-8_3.

4. Boehncke WH. Etiology and Pathogenesis of Psoriasis. Rheum Dis Clin North Am 2015;41:665-75.

5. Sonkoly E, Bata-Csorgo Z, Pivarcsi A, et al. Identification and characterization of a novel, psoriasis susceptibilityrelated noncoding RNA gene, PRINS. J Biol Chem 2005;280:24159-67.

6. Yan S, Xu Z, Lou F, et al. NF- B-induced microRNA-31 promotes epidermal hyperplasia by repressing protein phosphatase 6 in psoriasis. Nat Commun 2015;6:7652.

7. Qiao M, Li R, Zhao X, et al. Up-regulated IncRNAMSX2P1 promotes the growth of IL-22-stimulated keratinocytes by inhibiting miR-6731-5p and activating S100A7. Exp Cell Res 2018;363:243-54.

8. Geller S, Xu H, Lebwohl M, et al. Malignancy Risk and Recurrence with Psoriasis and its Treatments: A Concise Update. Am J Clin Dermatol 2018;19:363-75. 
9. Michalek IM, Loring B, John SM. A systematic review of worldwide epidemiology of psoriasis. J Eur Acad Dermatol Venereol 2017;31:205-12.

10. Ding X, Wang T, Shen Y, et al. Prevalence of psoriasis in China: a population-based study in six cities. Eur J Dermatol 2012;22:663-7.

11. Ouyang W. Distinct roles of IL-22 in human psoriasis and inflammatory bowel disease. Cytokine Growth Factor Rev 2010;21:435-41.

12. Shirakata Y. Regulation of epidermal keratinocytes by growth factors. J Dermatol Sci 2010;59:73-80.

13. Nedoszytko B, Sokołowska-Wojdyło M, RuckemannDziurdzińska K, et al. Chemokines and cytokines network in the pathogenesis of the inflammatory skin diseases: atopic dermatitis, psoriasis and skin mastocytosis. Postepy Dermatol Alergol 2014;31:84-91.

14. Kim J, Krueger JG. The immunopathogenesis of psoriasis. Dermatol Clin 2015;33:13-23.

15. Georgescu SR, Tampa M, Caruntu C, et al. Advances in Understanding the Immunological Pathways in Psoriasis. Int J Mol Sci 2019;20:739.

16. Wang X, Arai S, Song X, et al. Induced ncRNAs allosterically modify RNA-binding proteins in cis to inhibit transcription. Nature 2008;454:126-30.

17. Atianand MK, Fitzgerald KA. Long non-coding RNAs and control of gene expression in the immune system. Trends Mol Med 2014;20:623-31.

18. Tsoi LC, Iyer MK, Stuart PE, et al. Analysis of long noncoding RNAs highlights tissue-specific expression patterns and epigenetic profiles in normal and psoriatic skin. Genome Biol 2015;16:24.

19. Yan J, Song J, Qiao M, et al. Long noncoding RNA expression profile and functional analysis in psoriasis. Mol

Cite this article as: Wang Y, Zhu J, Xu J, Du J, Lu X. The long non-coding RNA and mRNA expression profiles in keratinocytes from patients with psoriasis vulgaris. Ann Palliat Med 2021;10(8):9206-9214. doi: 10.21037/apm-21-2046
Med Rep 2019;19:3421-30.

20. Széll M, Danis J, Bata-Csörgő Z, et al. PRINS, a primatespecific long non-coding RNA, plays a role in the keratinocyte stress response and psoriasis pathogenesis. Pflugers Arch 2016;468:935-43.

21. Szegedi K, Göblös A, Bacsa S, et al. Expression and functional studies on the noncoding RNA, PRINS. Int J Mol Sci 2012;14:205-25.

22. Qiao H, Li Y, Feng C, et al. Nap1l1 Controls Embryonic Neural Progenitor Cell Proliferation and Differentiation in the Developing Brain. Cell Rep 2018;22:2279-93.

23. Yan Y, Yin P, Gong H, et al. Nucleosome Assembly Protein 1-Like 1 (Nap111) Regulates the Proliferation of Murine Induced Pluripotent Stem Cells. Cell Physiol Biochem 2016;38:340-50.

24. Chen Z, Gao W, Pu L, et al. PRDM8 exhibits antitumor activities toward hepatocellular carcinoma by targeting NAP1L1. Hepatology 2018;68:994-1009.

25. Cevik RE, Cesarec M, Da Silva Filipe A, et al. Hepatitis C Virus NS5A Targets Nucleosome Assembly Protein NAP1L1 To Control the Innate Cellular Response. J Virol 2017;91:e00880-17.

26. Huang Y, Xiang B, Liu Y, et al. LncRNA CDKN2BAS1 promotes tumor growth and metastasis of human hepatocellular carcinoma by targeting let-7c-5p/NAP1L1 axis. Cancer Lett 2018;437:56-66.

27. Tay Y, Rinn J, Pandolfi PP. The multilayered complexity of ceRNA crosstalk and competition. Nature 2014;505:344-52.

28. Wang K, Long B, Jiao JQ, et al. miR-484 regulates mitochondrial network through targeting Fis1. Nat Commun 2012;3:781.

(English Language Editor: J. Teoh) 